\title{
Ralf Hohlfeld Qualität in Quoten? Der öffentlich-rechtliche Rundfunk in der Evaluations-Gesellschaft
}

Nach einer gewissen Phase der Stagnation leben derzeit die Bemühungen innerhalb der öffentlich-rechtlichen Funkhäuser wieder auf, die eigenen Programme kritisch zu bewerten. Auch und gerade bei den Praktikern setzt sich allmählich die Einsicht durch, daß es bestimmter qualitativer Sollvorgaben bedarf, um einen Sendeplatz zu definieren. ${ }^{1}$ Es herrscht Einigkeit darüber, daß Einschaltquoten alleine sich nicht dazu eignen, zuverlässige Aussagen über die Leistungsfähigkeit von Fernsehanbietern zu treffen. ${ }^{2}$ "Die eindimensionale Bewertung nach der Einschaltquote greift bei einem anspruchsvollen öffentlich-rechtlichen Programm zu kurz", befindet BR-Intendant Albert Scharf. ${ }^{3}$ Mit der Überprüfung durch anerkannte Leistungsindikatoren könnte, so die Überlegung innerhalb und außerhalb der Rundfunkanstalten, ein Gegengewicht zum Diktat der rein quantitativ ausgerichteten Zuschaueranteils-Quote geschaffen werden. Anhand von Leistungsindikatoren ermittelte "Qualitätsquoten" ließen sich unter Umständen zu einer öffentlichkeitswirksamen Gegenwährung zu den klassischen, nur auf die Akzeptanz bezogenen Reichweiten entwickeln, so vermuten und hoffen insbesondere die Sympathisanten des öffentlich-rechtlichen Systems. ${ }^{4}$

Als Dienstleister im weitesten Sinne kann sich auch der öffentliche Rundfunk in Deutschland nicht dem allgemeinen Trend zum Quality Management verweigern. Unter diesem Schlagwort evaluieren serviceorientierte Unternehmen seit einigen Jahren ihre Leistungskataloge: Ob PR-

1 Aus diesem Grund haben die zur Group of European Audience Researcher (GAER) zusammengeschlossenen europaischen Publikumsforscher das Thema 'performance indicators' auf die Tagesordnung gesetzt. Vgl. Diem, Peter: Leistungsindikatoren für den 8 ffentlich-rechtlichen Rundfunk. Versuch einer mehrdimensionalen Operationalisierung der Qualităt von Fernsehsendungen. In: Media Perspektiven 2/94, S. 67-71.

2 Vgl. Oehmichen, Ekkehart: Qualität im Fernsehen aus der Zuschauerperspektive. Ansätze praxisorientierter Forschung. In: Media Perspektiven, 1/93, S. 16-20, hier S. 16.

3 Albert Scharf, zitiert in: Bayerischer Rundfunk: Zusätzliches Management-Instrument: BR testet neue Programmwert-Kennziffern. Pressestelle des Bayerischen Rundfunks, Info 4/97, S. 1-2.

4 Vgl. Hömberg ruft nach "Qualitätsquoten". Tutzinger Tagung war sich einig: TV-Kultur ist Domăne der Öffentlich-Rechtlichen. In: epd medien, Nr. 74. vom 23. September 1998, S. 18f. Vgl. ebenfalls Scheuermann, Silke: An der Imbißbude. Eine Tutzinger Tagung sucht nach Qualitalt im Rundfunk. In: FAZ, Nr. 223, vom 25. September 1998, S. 42. 
Agentur oder Hotelkette, ob Bankunternehmen oder Fluggesellschaft: Überall wird - vor allem in Hinsicht auf die Aus- und Weiterbildung des Personals - nach einem Prüfsiegel gestrebt, meist in Gestalt der Zertifizierung nach ISO 9000ff. Verfolgt man die aktuellen Debatten in den Rundfunkanstalten, liegt der Schluß nahe, daß auch im Rundfunk, zumindest in dem Teil, der dem Public Service zugerechnet werden kann, die Qualitäts- und Leistungstransparenz das Gebot der Stunde ist. Die Gründe liegen auf der Hand: Die Komplexität der Informationsgesellschaft verursacht immer folgenreichere Evaluationszwänge. Um sich von den konkurrierenden Kommunikationsanbietern abzusetzen, muß ein Medienunternehmen seine Leistungen transparent machen. Dazu kann es sich entweder der internen oder der externen Evaluation bedienen. Die Selbstevaluation ist häufig ein Merkmal öffentlicher Träger. ${ }^{5}$ Anstrengungen zur Bewertung von Medienangeboten, wie sie sich z.B. in der Schaffung einer Stiftung Medientest ${ }^{6}$ äußern, sind Reaktionen auf die "neue Unübersichtlichkeit"7 des Mediensystems mit ihrer kaum noch zu überblickenden Fülle von General Interest- und Special Interest-Angeboten. Sie dienen ebenso als Navigationshilfen im Mediendschungel wie alle anderen Formen der Medienbewertung und Medienkritik.

Die zunehmende Undurchschaubarkeit des Medien-Dickichts regt auf natürliche Weise den Drang an, Unterschiede zu machen, Medienkommunikation auf ihre Beschaffenheit hin zu prüfen. Zwischen dem Mediensystem und seiner Referenzdisziplin, der Kommunikationswissenschaft, verläuft die Diskussion um Qualität deshalb fast parallel. Während sich die Kommunikationswissenschaft aber kaum einmal traut, übergreifende Maßstäbe expressis verbis $z \mathfrak{u}$ formulieren, weil der funktionalistische

5 Unter Evaluation ist hier mit Suchman der Prozeß der Beurteilung eines Produktes, Prozesses oder Programmes zu verstehen. Vgl. Edward A. Suchman: Evaluative Research. New York 1967. Im Falle der Sendungserfolgskontrolle handelt es sich um eine reine Produkt-Evaluation, die grundsätzlich von internen wie extemen Gutachtern durchgeführt werden kann. Zu den Vor- und Nachteilen beider Modi vgl. Schröter, Jens: Journalisten im Labor. Evaluation der European Initiative for Communicators of Science (EICOS). Diplomarbeit am Studiengang Journalistik der Katholischen Universität Eichstätt 1999, besonders S. 14-25.

6 Vgl. Krotz, Friedrich: Zur Konzeption einer Stiftung Medientest. In: Rundfunk und Fernsehen, 2/96, S. 214-229. Anfang Dezember 1998 berieten die Ministerpräsidenten der Länder über die Gründung einer solchen Stiftung, die wie die gleichnamige Stiftung Warentest arbeiten soll. Vgl. Länder planen Stiftung Medientest. Regelmäßig erscheinende Publikation soll informieren. In: Süddeutsche Zeitung, Nr. 276 vom 30. November 1998, S. 19.

7 Meckel, Miriam: Die neue Unübersichtlichkeit. Zur Entwickung des FormatFernsehens in Deutschland. In: Rundfunk und Fernsehen, $45 \mathrm{Jg} .1997$, Heft 4, S. 475-485. 
Mainstream, das „dominant paradigma“, dies verbietet, ${ }^{8}$ lastet seit der Dualisierung des Rundfunks ein verstärkter Handlungsdruck auf den öffentlich-rechtlichen Rundfunkanstalten.

Dabei befindet sich der öffentliche Rundfunk in einer doppelten Wettbewerbs- und Legitimationszange. Zum einen muß er den gesellschaftspolitisch auferlegten Aufgaben und Anforderungen mehr denn je entsprechen. Die Qualitätsprüfung ist erst sehr spät, in der aufkommenden Konkurrenzsituation, ein Thema geworden, obwohl Qualität als Zielvorgabe in den Programmgrundsätzen festgeschrieben steht. Da jüngst einige wissenschaftliche Untersuchungen zur Konvergenz im dualen Fernsehsystem dazu beitrugen, die programmlich-inhaltlichen Entsprechungen des öffentlich-rechtlichen Sonderstatus anzuzweifeln, ist die FunktionsErfüllung aber nicht mehr unumstritten. ${ }^{9}$

Zudem sind die dem öffentlich-rechtlichen Rundfunk aufgetragenen Aufgaben meist in widerspenstige und sperrige Rechtsbegriffe gepreßt worden, so daß es ohnehin schon immer notwendig gewesen wäre, die Werte und juristischen Präskriptionen wie Grundversorgung, Gemeinwohlorientierung, meritorische Güter, Medium- und Faktor-Formel, Integrationsfunktion usw., die fraglos den einklagbaren Gegenwert zum Gebührenprivileg bilden, mit Inhalten zu füllen. Doch erst unter den Legitimationszwängen, die Mitte der 80er entstanden, ist in den Rundfunkanstalten die Einsicht erwacht, daß unbestimmte Rechtsbegriffe wie die Grundversorgung allein nicht mehr als Ausweis für ein instrumentelles Rundfunkverständnis ausreichen; vielmehr müßten auch die Inhalte herangezogen werden, um Qualitätsmaßstäbe zu erzeugen. Überspitzt formuliert fällt dabei dem öffentlich-rechtlichen Rundfunk die undankbare Aufgabe zu, Dolmetscher seiner eigenen Leistungen zu sein. Öffentlich-

Vgl. McQuail, Denis: Mass Communication Theory. An Introduction, London et al. 1994.

9 Vgl. Bruns, Thomas/Marcinkowski, Frank: Konvergenz Revisited. Neue Befunde zu einer älteren Diskussion. In: Rundfunk und Fernsehen, 44. Jg. 1996, Heft 4, S. 461-478; Marcinkowski, Frank/Bruns, Thomas: Politische Magazine im dualen Fernsehen. Probleme einer Unterscheidung. In: Schatz, Heribert (Hrsg.): Fernsehen als Objekt und Moment sozialen Wandels. Faktoren und Folgen der aktuellen Verändenungen des Fernsehens. Opladen 1996, S. 255-286; Merten, Klaus: Konvergenz der deutschen Fernsehprogramme. Eine Langzeituntersuchung 1980 bis 1993. Münster-Hamburg 1994; Pfetsch, Barbara: Konvergente Femsehformate in der Politikberichterstattung? Eine vergleichende Analyse öffentlich-rechtlicher und privater Programme 1985/1986 und 1993. In: Rundfunk und Fernsehen 1996, 44. Jg 1996, Heft 4, S. 479-498. Als Gegenposition vgl. Krüger, Udo Michael: Zum Stand der Konvergenzforschung im Dualen Rundfunksystem. In: Walter Klingler/Gunnar Roters/Oliver Zollner (Hrsg.): Fernsehforschung in Deutschland. Themen - Akteure Methoden. Baden-Baden 1998, S. 151-184. 
rechtlicher Rundfunk muß zunehmend gesellschaftlich vermitteln, was seine Besonderheit ausmacht, insbesondere in einer Zeit, in der Zuschauerschaften heranwachsen, die nicht ausschließlich öffentlich-rechtlich sozialisiert wurden, die in ihm schlicht ein beliebiges, unspezifisches Angebot unter vielen anderen sehen. Soviel dürfte feststehen: Die Indifferenz ist der Todfeind des öffentlichen Rundfunks, weil sie seinen Legitimationsvorschuß untergräbt und seinen verfassungsrechtlich gestützten Status aushöhlt.

Weder die Medien- und Kommunikationspolitik noch die Wissenschaft haben den Öffentlich-Rechtlichen bislang nachhaltig geholfen, den Legitimationsdruck zu mindern. Einzig das Bundesverfassungsgericht hat immer wieder die Notwendigkeit des Wertegarants öffentlich-rechtlicher Rundfunk betont, ohne allerdings präzise Vorgaben für die Funktionserfüllung zu machen. Ohne Schützenhilfe muß der öffentliche Rundfunk deshalb selbst dafür sorgen, daß seine Existenzberechtigung im Bewußtsein der Gesellschaft verankert bleibt. Wem niemand zur Seite springt, der muß selbst initiativ werden: Auch unter diesem Gedanken sind die jüngsten Aktivitäten zu sehen, die die übliche Praxis der Programmbewertung, aus dem Bauch heraus, ablösen wollen.

Der zweite Druckfaktor für Evaluationsbestrebungen ist ein mediensystem-immanenter: Er ist kompetetiver Natur und wirkt dabei gleichzeitig nach innen, in die Redaktionen, und nach außen, auf den gesamten Fernsehmarkt. Der Wettbewerbszwang des dualen TV-Systems führt dazu, programmplanerische Entscheidungen nachvollziehbarer zu machen und auf eine rationale Basis zu stellen. Deshalb muß das eigene Programm bis ins Detail bewertbar und die Planung transparent gemacht werden, und zwar sowohl im Hinblick auf interne Vergleichbarkeit (benchmarking) mit ähnlichen Programmen aus dem eigenen Haus als auch in bezug auf die extern orientierte Marktfähigkeit. Den Programmverantwortlichen stellt sich die Frage: Welche publizistischen und ReputationsChancen besitzt die einzelne Sendung im Fernsehsystem?

Selbstverständlich spielen auch Kosten- und Effizienzerwägungen seit Ende der 80er Jahre eine zunehmend größere Rolle. Zusammengenommen münden diese Überlegungen in das Erfordernis einer Sendungserfolgskontrolle als zentrales Steuerungsinstrument der Programmplanung. Sofern man prinzipiell von der Qualität der eigenen Produktionen überzeugt ist, liegt es nahe, intern zu entwickelnde Erfolgsparameter mit außenwirksamen Leistungsindikatoren zu verknüpfen. So gesehen wären unter Qualitätsquoten Meßinstrumente zu verstehen, die zwischen externer Leistungsschau und internem Controlling oszillieren. 


\section{Anmerkungen zur Meßbarkeit von Qualität}

"Identifying the undefinable" - so überschrieb Timothy Leggatt seinen Essay über das zum Scheitern verurteilte Unterfangen, Programmqualität zu vermessen. ${ }^{10}$ An Bemühungen dazu mangelt es freilich nicht, nur laufen die meisten Vorstöße Gefahr, aus subjektiver Sicht Normen für den Geschmack zu definieren. ${ }^{11}$ Mit dem Versuch, „einen Pudding an die Wand zu nageln"12, vergleicht Stephan Ruß-Mohl die Ansätze zur journalistischen Qualitätsbestimmung. Es ist ein erkenntnistheoretischer Allgemeinplatz, daß die qualitative Beurteilung subjektiv ist. „Es ist immer nur eine Annäherung an ein Ideal möglich, wie auch immer die qualitative Bewertung operationalisiert wird." 13 Gleichwohl herrscht Einigkeit darüber, daß Qualitätskriterien der wissenschaftlichen Analyse zugänglich sind. . $^{13 \mathrm{a}} \mathrm{Um}$ aber nicht von vornherein resignativ im Anekdotischen $z u$ verbleiben, müssen Überlegungen zur Objektivierung des Subjektiven angestellt werden.

Vorausgesetzt, daß Wolf Schneiders lakonisches Diktum "Qualität kommt von Qual" nicht der Ausgangspunkt einer ernsthaften Beschäftigung sein kann, beschreibt der Begriff Qualität aus der Perspektive der Wissenschaft zunächst lediglich den Zustand eines publizistischen Produkts anhand von wahrnehmbaren Eigenschaften. ${ }^{14}$ Qualität ist vorläufig nichts anderes als eine wertfreie Aussage über die "Wie-Beschaffenheit", Qualität bedeutet vor-empirisch nicht automatisch "gut". Uwe Kammann spricht in bezug auf den Rundfunk von einer nach oben und unten offenen Güteskala: „Denn Qualitäten haben alle Programme. Es kommt nur darauf an, welche." 15

$10 \mathrm{Vgl}$. Leggatt, Timothy: Identifying the undefinable: an essay on approaches to assessing quality in television in the U.K. In: Studies of Broadcasting 27, 1991, S. 121-156.

11 Merten 1994 (Anm. 9), S. 21.

12 Ruß-Mohl, Stephan: Der I-Faktor. Qualitätssicherung im amerikanischen Journalismus - Modell für Europa? Zürich 1994, S. 94.

13 Schlote, Axel/Latzel, Peter: Bewertung von Fernsehsendungen. Chancen und Probleme einer Objektivierung von Programmentscheidungen. In: Walter Klingler/ Gunnar Roters/Oliver Zollner (Hrsg.): Fernsehforschung in Deutschland. Themen Akteure - Methoden. Baden-Baden 1998, S. 815-837, hier S. 819.

13a Vgl. Wilke, Jürgen: Was heißt journalistische Qualitat? Auch ein Versuch zur Be. stimmung ihrer Kriterien. In: Duchkowitsch, Wolfgang et al. (Hrsg.): Journalismus als Kultur. Analysen und Essays. Opladen, Wiesbaden 1998, S. 133-142.

14 Vgl. Hohlfeld, Ralf: Fernsehprogrammanalyse. Formen, Einsatzmöglichkeiten und Reichweite. In: Walter Klingler/Gunnar Roters/Oliver Zollner (Hrsg.): Fernsehforschung in Deutschland. Themen - Akteure - Methoden. Baden-Baden 1998, S. 197 224. 
Auf der Suche nach den eigenschaftentragenden Indikatoren für Qualität versperren gewichtige erkenntnistheoretische Einwände den Weg. Die Selektion der Eigenschaften, die zu beschreiben sind, ist ein erster normativ-subjektiver Akt, denn es handelt sich um eine Zuweisung, die von außen an die Kommunikation herangetragen wird. Da diese Selektion jeder Analyse notwendigerweise vorausgehen muß, hat die Untersuchung von Qualität traditionellerweise einen doppelten normativen Bezug.

Wenn es darum geht, festgelegte Erfordernisse zu erfüllen, müssen Maßstäbe explizit angelegt werden. Aus empirischer Sicht entspricht Qualität einem Merkmal, das Standards genügt, die auf zentralen Werten und Normen beruhen. Letztere wiederum sind Fremdzuschreibungen. In diesem Sinne verstanden, kann man Qualität schlechterdings nicht seiner normativen, subjektiven Natur berauben. Für eine empirische Programmforschung bedeutet dieses subjektive Moment, daß die Lösung schon in der Problemstellung mitgeliefert wird. Es müssen möglichst viele Objektbereiche aus der Umwelt des Untersuchungsfeldes in die Analyse einbezogen werden. Es gilt, Qualität gewissermaßen von außen her $\mathrm{zu}$ "objektivieren“. Voraussetzung dafür ist, die Beschaffenheit der medial vermittelten Kommunikation $\mathrm{zu}$ beschreiben und die normierend einwirkenden Maßstäbe - welche Facetten der Beschaffenheit interessieren? - offenzulegen. Dies geschieht analog zum wissenschaftstheoretischen Objektivitätsproblem, das nach Popper dadurch gelöst wird, daß die Objektivität der wissenschaftlichen Sätze, allgemein gesprochen, durch die intersubjektive Nachprüfbarkeit gewährleistet wird. Es müssen demzufolge externe Bezugspunkte für eine Objektivierung gefunden werden, deren Relevanz zu begründen ist.

In diesem Sinne ist der Qualitätsbegriff variabel und hinsichtlich jedes zu beurteilenden Gutes neu erklärungsbedürftig. Er hat stets eine Ausrichtung, da er von der Zuweisung durch eine Beobachterrolle, einem spezifischen Interesse und einem Zweck abhängt. Gerichtet ist er auf Normen und Werte, schlechtestenfalls auf Gesinnung und Ideologie. Qualität in diesem Sinne ist teleologisch, auf Zielvorstellungen hingeordnet. Die Besetzung mit positivem oder negativem Wert, die Zuweisung einer Richtung, ist erst die zweite - intersubjektiv nachvollziehbar zu machende - Selektion: In welcher Weise ist eine Eigenschaft funktional für ein definiertes Ziel?

„Läßt sich über Qualität streiten?"16 lautet die rhetorische Frage von

15 Kammann, Uwe: Kifu-Tagebuch: Wie Qualitätsprogramme à la „Fitz“ behandelt werden. In: epd-Kirche und Rundfunk, Nr. 77 vom 2. Oktober 1996, S. 2.

16 Vgl. Weiß, Ralph: Läßt sich liber Qualităt streiten? Versuche in der Kommunikati- 
Ralph Weiß. Qualität, verstanden als Summe der Merkmale und Eigenschaften, die ein bestimmtes Gut tauglich machen, dem subjektiven Geschmack zu entsprechen - darin liegt die Chance, den Qualitätsbegriff zu objektivieren. Mit diesem Ansatz ist Weiß nicht weit entfernt von Max Webers Ausführungen über die charismatische Herrschaft: „Wie die betreffende Qualität von irgendeinem ethischen, ästhetischen oder sonstigen Standpunkt aus, objektiv' richtig zu bewerten sein wïrde, ist natürlich dabei begrifflich völlig gleichgültig: darauf allein, wie sie tatsächlich von den charismatisch Beherrschten, den ,Anhängern' bewertet wird, kommt es an. ${ }^{17}$ Sowenig wie Charisma eine Eigenschaft ist, die jemand oder etwas an sich hat, ist auch Qualität kein Merkmal aus sich selbst heraus, sondern eine Zuschreibung. Qualität ist das, was die Zuschauer funktionell dafür halten, ohne es zu wissen. Hier muß Qualitätsforschung ansetzen. Festzuhalten bleibt indes: Qualitätsurteile haben naturgemäß eine dominante subjektive Komponente. Um die Urteile an objektivierbaren Maßstäben anszurichten, maß man „ihre Voraussetzungen explizieren, ihren Gehalt analysieren und ihre Verbreitung und Akzeptanz prüfen." $17 \mathrm{a}$

\section{Chancen und Risiken der qualitativen Programmbewertung}

Unter dem Arbeitstitel Programmwert-Kennziffer (PWKZ) kursieren in den Rundfunkanstalten seit circa 1993 vielfältige Aktivitäten, die nicht alle einen identischen Bezugspunkt besitzen. Der kleinste gemeinsame Nenner ist die Auslotung des Wertes oder Erfolges einer Sendung. Ob dieser allerdings einem wirtschaftlichen Maßstab folgt, d.h. nur auf die Kosten von Material und Personal reduziert bleibt, ob der publizistische Erfolg damit gemessen werden soll, oder ob gar der immaterielle Wert sichtbar gemacht werden soll, ist von Fall zu Fall verschieden. Vorläufer der Ermittlung des inhaltlichen Wertes war in jedem Fall die von der Kommission zur Ermittlung des Finanzbedarfs (KEF) angeregte Errechnung der durchschnittlichen Produktions- und Personalkosten je Sende-

onswissenschaft zur Objektivierung des Qualitätsbegriffs. In: Hartmut Weßler et al: (Hrsg.): Perspektiven der Medienkritik. Die gesellschaftliche Auseinandersetzung mit offentlicher Kommunikation in der Mediengesellschaft. Opladen 1997, S. 185-199.

17 Zitiert nach Podak, Klaus: Charisma - das will ich sehen. Warum nur manche Menschen durch die Medien zu strahlenden Helden werden können. In: Süddeutsche

Zeitung, Nr. 269, vom 21./22. November 1998, S. 18.

17a Wilke 1998, S. 134 (Anm. 14). 
minute, der Sendeminutenkosten je Programmart und Ressort und der Personalkosten pro Festangestelltem bei den Öffentlich-Rechtlichen. ${ }^{18}$ Ebenso wie die Zuschauerreichweite ignorieren jedoch die auf Wirtschaftlichkeit zielenden Parameter die - im Vergleich zu privat-kommerziellen Sendern - unterschiedlichen Vorgaben, Ziele und Funktionen. „Der Nährwert wird ignoriert." 19 Und weitaus gravierender: Es wird die Möglichkeit vernachlässigt, mit den öffentlich-rechtlichen Pfunden $z u$ wuchern. Seit die Anstalten zur Einsicht gekommen sind, daß sich aus dem programmlichen Nährwert auch ein Mehrwert erzielen läßt, nehmen die Bemühungen $z u$, den diffizilen Begriff der Qualität zu operationalisieren und gemeinsam mit den Indikatoren für Akzeptanz und Wirtschaftlichkeit in einen Index zu überführen. Die strategische Idee einer derartig komplexen Programmwert-Kennziffer kommt jedoch nicht aus den Reihen der Programmacher, sondern von der Spitze, den Programmverantwortlichen, dem Management.

Die strategische Bedeutung einer solchen kombinierten Maßzahl, die quantitative und qualitative Kriterien zusammenführt, läßt sich in viele Facetten zerlegen. Intern wird der Nutzen vor allem in der Sendungserfolgskontrolle gesehen. Für jeden Sendeplatz wird in bezug auf die Reichweite, die Kosten und die Qualität eine festumrissene Zielvorgabe gemacht, die hilft, Schwächen abzustellen, Fehler zu vermeiden und - gemessen am Aufwand - Leistungen zu optimieren. Die Beobachtung langfristiger Entwicklungen anhand von Maßzahlen stützt gravierende Entscheidungen bei der längerfristigen Programmplanung. Auch das Motivationselement, das der Transparenz von Erfolgskontrollen innewohnt, darf nicht unterschätzt werden. Konkurrenz belebt das Geschäft; die Vergleichbarkeit mit strukturähnlichen Programmen kann dazu beitragen, daß Engagement zu befördern und die Mitarbeiter zu höheren Leistungen anzuspornen.

Selbstverständlich treffen Maßnahmen, die zum internen Controlling gerechnet werden können, in den über Jahrzehnte von Gängelung und Beaufsichtigung freien Rundfunkanstalten auf Vorbehalte und Widerstände. Verwiesen wird in der Regel auf das Mißbrauchspotential eines solchen Transparenz-Modells, das scheinbar objektive Argumente zur Absetzung mißliebiger Sendungen liefert. Folgende Sorgen und Ängste werden entsprechend - gerade von altgedienten Mitarbeitern - artikuliert: - Die Kontrolle durch das eigene Haus ist eine ungewohnte Situation. Besonders dann, wenn den Controlling-Maßnahmen der Geruch anhaftet, in den Stabstellen der Intendanz angesetzt worden zu sein.

18 Vgl. Schlote/Latzel 1998 (Anm. 13).

19 Ebenda, S. 816. 
Wer je in einer öffentlich-rechtlichen Anstalt gearbeitet hat, kennt die Skepsis und Berührungsängste gegenüber jeder Form von Kontrollmaßnahmen durch das Management. Vor allem dann, wenn zu diesem Zweck eine externe Unternehmensberatung eingeschaltet wird, die nach Rationalisierungsreserven sucht.

- Kritik an derartigen internen Steuerungsinstrumenten speist sich auch aus simplem Besitzstandsdenken: Als Instrument einer apriori-Programmgestaltung eingesetzt, können durch die Sendungserfolgskontrolle Projekte im Vorfeld umgestrickt oder gekippt, kann Einfluß auf die Autonomie der Redaktionen genommen werden. Dahinter steckt die Furcht, fixierte Vorgaben nicht erfüllen zu können.

- Besonders scharf ist die Ablehnung gegenüber einer Bewertungsinstanz, die nicht aus der journalistischen Praxis stammt. NichtPraktikern, die mit den Produktionbedingungen nicht vertraut sind, wird die Beurteilungskompetenz schlichtweg abgesprochen. Praktiker vertreten den Einwand, Handwerkliches könne niemals durch externe Berater bewertet werden.

- Auch der Kunstfaktor bei fiktionalen Sendungen, der sich schwer erfassen läßt, wird als Gegenargument hervorgebracht: Ästhetische Verfremdungsmittel lassen sich unter Umständen fälschlicherweise als mangelhafte Bildqualität deuten. Verwiesen wird immer wieder auf das Mißbrauchspotential eines solchen Modells.

- Zum Tragen kommt auch ein Moment, das der Deutschland-Radio Intendant, Ernst Elitz, als „öffentlich-rechtliche Entitäten“ 20 bezeichnet: Das Bewußtsein, als Kulturschaffende mit allen Freiheiten ausgestattet $\mathrm{zu}$ sein und sich per se einer auf Verallgemeinerung zielenden Beurteilung entziehen zu können. Das publizistische Produkt als solches sträubt sich allgemein gegen die wissenschaftliche Beurteilung. Dahinter steht als Motiv die Furcht, beim Durchleuchtetwerden Herrschaftswissen einzubüßen.

Gleichwohl erkennen immer mehr Medienpraktiker die strategischen Einsatzmöglichkeiten. Pragmatiker wie Elitz, die über die selbstreferentielle Qualitätsdefinition in den öffentlich-rechtlichen Häusern klagen, sind keine Seltenheit mehr. Und selbst aus den Kultur-Redaktionen, früher der Hort der Selbstbezüglichkeit schlechthin, ergehen unterdessen Aufforderungen an die Medienforschung, die eigenen Programme einer kritischen Bewertung zu unterziehen. Dort begreift man die Sen-

20 So Ernst Elitz in einem Vortrag zum Thema "Qualităt, Quark und Quote", den der Deutschland-Radio-Intendant anlaßlich der Eichstätter Medientage im Rahmen der Veranstaltungsreihe „Journalistisches Kolloquium“ am 26. November 1998 hielt. 
dungsbeurteilung nicht mehr als Gefahr der Kontrolle im Vorfeld, sondern als Möglichkeit, quotenschwache Redaktionen systematisch wieder aufzubauen.

Die externe Wirkung der Programmwert-Kennziffern wird in den Anstalten selbst noch vergleichsweise geringgeschätzt. Ganz anders die (kritischen) Sympathisanten und Förderer des öffentlichen Rundfunks, die nachgerade auf eine Leistungsschau drängen.

Ungeachtet der Hemmnisse und Widerstände intra muros fordern die Förderer des öffentlich-rechtlichen Systems einen weitaus offensiveren Umgang mit der Programm- und Sendungsbewertung. ${ }^{21}$ Vertrauend auf einen wie auch immer gearteten Qualitätsvorsprung, der sich auch in der jüngeren Vergangenheit allen Konvergenzvermutungen zum Trotz gehalten haben mag, sehen sie darin eine Möglichkeit, den privaten Wettbewerbern eine Disziplin aufzuzwingen, in der sie kaum eine Gewinnchance haben. Auf diese Weise kann der öffentlich-rechtliche Rundfunk ein Stück Definitionsmacht zurückerobern, die er in der auf Akzeptanz und Quote verengten medien- und gesellschaftspolitischen Diskussion schon weitgehend verloren hatte. Inwieweit ein gewinnorientierter privat-kommerzieller Rundfunk einen derartigen Schlagabtausch anzunehmen bereit ist, dürfte zunächst von sekundärem Interesse sein. Zuerst, so fordern die Sympathisanten, muß der Ball ins Spiel gebracht werden. Inwieweit dann ein Kräftemessen zustande kommt, ist eher eine Frage geschickter PR-Arbeit.

Die Idee einer gemeinsamen qualitativen Währung zwischen öffentlich-rechtlichen und privaten Rundfunkanbietern ist nicht gerade neu. Die Notwendigkeit eines solchen Vergleichs, insbesondere als komparatistisches Mittel der Programmaufsicht und -kontrolle über den Privatfunk, ist immer wieder in die medienpolitische Debatte eingebracht worden. ${ }^{22}$ Man brauche eine verbindliche „Währung, auf die sich alle Programmveranstalter gemeinsam beziehen, weil sie sich darauf verständigt haben", so der Direktor der Landesanstalt für Rundfunk Nordrhein-

21 Auf einer Veranstaltung des Münchner Arbeitskreises offentlicher Rundfunk (MAR) forderte deren Sprecher, der Joumalistik-Professor Walter Homberg, Einschaltquotenmessungen durch inhaltliche und formale Bewertungen zu ergänzen, "wozu die Einführung von Programmwert-Kennziffern ein sinnvoller Schritt sein könnte“. Zitiert nach N.N.: Hömberg ruft nach "Qualitätsquoten". Tutzinger Tagung war sich einig: TV-Kultur ist Domäne der Öffentlich-Rechtlichen. In: epd medien, Nr. 74. vom 23. September 1998, S. $18 f$.

22 Und in Ansätzen auf programmstruktureller Ebene auch umgesetzt worden. Vgl. zum Beispiel Weiß, Hans Jürgen/Trebbe, Joachim: Öffentliche Streitfragen in privaten Fernsehprogrammen. Zur Informationsleistung von RTL, SAT.1 und Pro Sieben. Opladen 1994. 
Westfalen, Norbert Schneider. ${ }^{23}$ Mit seinen Kollegen von der Direktorenkonferenz der Landesmedienanstalten (DLM) hat Schneider eine Studie zur Entwicklung einer einheitlichen Methode der Programmbeobachtung in Auftrag gegeben. Das in Fragen der Programmforschung erfahrene Göttinger Institut für angewandte Kommunikationsforschung (GÖFAK) wird für das Auftragsvolumen von 270.000 Mark versuchen, einen einheitlichen Bewertungsstandard zu entwickeln. Man erhofft sich neben dem strukturellen Überblick über die Gesamtprogramm-Proportionen auch die Grundlage eines sicheren Urteils über die Entwicklung einzelner Fernsehprogramme, und zwar jenseits punktueller Spekulationen über die Gründe für zu spät gesendete Kulturberichterstattung, zunehmende Gewalt oder Verrohung der Gesprächskultur.

Bislang hat sich der Verband privater Rundfunk und Telekommunikation (VPRT) einem solchen Vielfaltsvergleich erfolgreich verweigert. Einige Versuche von ARD und ZDF, eine gemeinsame Programmforschung anzuregen, sind von den Privaten stets abgelehnt worden. Neuerdings mehren sich die Zeichen, daß hier ein Umdenken einsetzt. Von Desinteresse an verbindlichen Bewertungskriterien könne keine Rede sein. Allerdings wird in einem solchen Fall darauf verwiesen, daß man sich keinesfalls auf ein in öffentlich-rechtlicher Eigenregie entwickeltes Instrument einlassen würde. Manfred Neuber vom VPRT rechtfertigt die Verweigerungshaltung der privaten Anbieter mit dem Hinweis darauf, daß private Sender sich nicht mit einem Bewertungssystem messen lassen können, "das die ARD für sich geschaffen hat".24

\section{Projekte zur Ermittlung von Leistungsindikatoren}

\section{ARD unter Federführung des Bayerischen Rundfunk}

Der Bayerische Rundfunk startete im Sommer 1998 eine Pilotstudie "Qualitätsbewertung“, mit der ein Teil der Programmwert-Kennziffer berechenbar wird. Für den internen Einsatz ist ein Soll-Ist-Vergleich mit festen Vorgaben für jeden Sendeplatz geplant. Zwei wichtige Voraussetzungen mußten für die Installation der PKWZ gegeben sein: Die Ziffer muß genre-übergreifend berechenbar und bei allen Sendeplatzverantwortlichen akzeptiert sein. Unter Einbeziehung externer Experten und Fachwissenschaftler versuchte die hauseigene Medienforschung, die Eig-

23 Norbert Schneider, zitiert in: Der lange Weg zu einheitlicher Programmforschung. DLM.Studie soll allgemeinverbindliche Bewertung von Sendern ermöglichen. In: Frankfurter Rundschau, vom 7. Oktober 1998, S. 10.

24 Ebenda. 
nung von Beurteilungsindikatoren zu prüfen. Grundlage für die Beurteilungsindikatoren waren einige qualitative Gruppendiskussionen, die im Herbst 1997 mit Zuschauern und sogenannten Programmexperten durchgeführt wurden.

Es zeichneten sich bei den zehn, im Anschluß an die Ansicht exemplarischer Sendungen des BR erarbeiteten Kriterien vier Gruppen ab: Anspruchsbezogene Indikatoren, zuschauerbezogene Indikatoren, Kompetenzindikatoren und Konkurrenzindikatoren. Zur ersten Gruppe zählen der „Beitrag zur Programmidentität", die „Werthaltung“ und die "gesellschaftliche Relevanz". Als rezeptionsorientierte Qualitätsindikatoren wurden die „Zuschauernähe“, der „Informationswert" und der „Unterhaltungswert" isoliert. Faktoren der Kompetenz sind die „redaktionell handwerkliche Befähigung" und die "gestalterische Kompetenz", die die Umsetzung und die daraus resultierende Anmutung umfaßt. Zu den Konkurrenzindikatoren gehören schließlich der "Innovationswert", verstanden im Sinne einer Trendsetterfunktion, und die "Einzigartigkeit", der Besonderheitsfaktor Uniqueness oder Unique Selling Point (USP). Im Pilotprojekt wurden die Experten gebeten, jede Sendung anhand einer elfstufigen Skala von 0 bis $10 \mathrm{zu}$ bewerten, und zwar jeweils hinsichtlich aller zehn Qualitätsindikatoren.

Die solchermaßen ermittelten Qualitätsquoten gehen als ein Faktor unter vieren in die Berechnung der eigentlichen Programmwert-Kennziffer ein. Daneben stehen die Kosten (Aufwand), die Akzeptanz (Marktanteil) und die (Wieder-)Verwertbarkeit der Programme, also ihre Poolfähigkeit, ihre Chance auf Mehrfachverwertung, Weiterverkauf etc. Die Programmwert-Kennziffern sind wiederum in ein übergeordnetes System eingebettet, das Aussagen über die Erfüllung von Anforderungsmaßstäben geben soll. $\mathrm{Zu}$ diesem Zweck haben die Programmdirektoren der ARD schon 1994 erste Sendeplatzprofile festgelegt, in denen die Anforderungen für jede Sendung genau festgelegt sind. Obwohl das Beurteilungsschema sendungsübergreifend konzipiert wurde, wird der Soll-istVergleich nur für Sendungen gleicher Art vorgenommen.

\section{Schweizer Fernsehen DRS}

Auch das Schweizer Fernsehen DRS arbeitet mit dem Instrument der Sendungserfolgskontrolle. ${ }^{25}$ Unter dem Eindruck schwerwiegender Reichweiteneinbrüche und Marktanteilsverlusten wurde 1993 mit Hilfe der Erfolgskontrolle eine Bestandsaufnahme des eigenen Programms durchgeführt. Im Zusammenhang mit einer organisatorischen Neuord-

25 Vgl. Wildberger, Jürg: Das Instrument der Sendungserfolgskontrolle. Schweizer Fernsehen DRS: Mehr Zuschauernähe und schärferes Programmprofil. In: Media Perspektiven, 2/94. S. 63-66. 
nung (Verflachung der Hierarchien, Kompetenzzuwachs der Sendungsverantwortlichen) wurden alle Redaktionen auf die Einhaltung vereinbarter Ziele verpflichtet. Die pauschale Vorgabe lautete, Marktführer zu bleiben, ohne den Auftrag als Public Service Sender zu gefährden. Neben der Einschaltquote und den Kosten wurde als dritter Faktor der Sendungs-Erfolgsprüfung die Reputation ermittelt. Dies ist zwar ebenfalls eine qualitative Dimension des Erfolgs, die sich aber im Unterschied zu den übrigen Verfahren deutlich über die Grenzen der eigentlichen Qualität erstreckt. Sie wird über sechs Kriterien bestimmt: „Akzeptanz und Erfolg in der Zielgruppe“, "Attraktivität und Verständlichkeit der Sendung “" "Qualität der Sendung", "Vergleich der Sendung mit Sendungen auf Drittsendern“, „Resonanz in anderen Medien und in der Öffentlichkeit", „Renommee für die Sendung und das Unternehmen“.

Qualität ist hier also nur ein Faktor unter vielen, der zur Reputation beiträgt. Abgesehen davon, daß einige Kriterien nicht trennscharf sind, andere wiederum Merkmale vermengen, die wesensmäßig wenig miteinander zu tun haben (Attraktivität und Verständlichkeit), wird hier ein neuer Weg beschritten. Die Außenwirkung der Sendungen steht eindeutig im Vordergrund der Evaluation, ihre eigentliche Beschaffenheit tritt zurück, denn die Qualität, die wiederum zu einem Teil durch Zuschauer bewertet wird, geht letztlich nur mit einem Achtzehntel in die Berechnung des Erfolgs ein. Ausgangspunkt ,war also ein betriebswirtschaftliches Kalkiuil“. ${ }^{26}$

Die Beurteilungen werden von sogenannten Linienverantwortlichen vorgenommen: Redaktionsleiter und Abteilungsleiter vergeben je Kriterium eine Note von 1 = tief bis $5=$ hoch, die dann gemittelt werden zur Reputationsnote. Auf der Basis des Reputationswertes wird zusammen mit dem Marktanteil in einem ersten Schritt eine Sendungstypologie erstellt, die Sendungen von A (nur hoher Marktanteil) über B (hoher Marktanteil und hohe Reputation) und C (geringer Marktanteil aber hohe Reputation) bis D (niedriger Marktanteil bei geringer Reputation) klassifiziert. In einem zweiten Schritt werden dann jene Kosten, die der Redaktionsleiter selbst verantwortet, als Kosten pro Sendeminute mit dem Marktanteil und der Reputation verrechnet. Auf die Aggregation in eine Maßzahl wird verzichtet, statt dessen wird zur Veranschaulichung die Sendung in einer graphischen Abbildung positioniert. Mit diesem Modell wurde eine tiefgreifende Programmstruktur-Reform begleitet, an deren Ende von 60 Sendungen zwölf gestrichen und zehn renoviert wurden. Die Auswirkungen auf der Personalebene waren ähnlich krass: Von sieben Abteilungsleitern blieben am Ende drei übrig. 
3sat

Einen vollkommen anderen Weg hat 3 sat eingeschlagen. Die Programmbewertung ist, wie man das von einem Sender mit kulturellem Schwerpunkt erwarten kann, ganz auf die journalistische und redaktionelle Qualität abgestellt. Zusammen mit den Redaktionen entwickelte eine Arbeitsgruppe Programmcontrolling einen sieben Indikatoren umfassenden Kriterienkatalog mit dazu abgestimmten Gewichtungsfaktoren. Das Qualitätsprofil besteht aus "Imagekraft", „Originalität/Exklusivität“ (Faktor 2), "Themenrelevanz", „inhaltliche Vermittlung“, „formale Qualität", "publizistisches Echo" (Faktor 1) und "Zuschauerresonanz" (Faktor 0,5). Die Bewertungsskala gipfelt in der Maximalpunktzahl von 85 Punkten, von denen mindestens 50 erreicht werden müssen. Ökonomische Parameter fehlen vollständig, was durch die Programmphilosophie des Senders gerechtfertigt ist. Quasi außer Konkurrenz werden die Beurteilungswerte mit quantitativen Zuschauerdaten wie Einschaltquote und Marktanteil „angedickt“.

\section{Zweites Deutsches Fernsehen}

Auch das ZDF testet derzeit ein qualitatives Controlling seiner Sendungen. Anhand von Sendeplatzprofilen wird nach Maßgabe der Variablen Inhalt/Funktion, Form, Zielgruppe und Imagebeitrag eine Bestandaufnahme aller ZDF-Sendungen durchgeführt. Im Zusammenspiel zwischen Redaktion, Direktion und Programmplanung wird für jede Sendung eine Zielbestimmung in Form einer Soll-Vorgabe festgelegt, deren Nichterreichen unmittelbare Konsequenzen nach sich ziehen soll. Allerdings erprobt man hier bislang die Akzeptanz im eigenen Haus und sammelt sukzessive Erfahrungen mit solchen Steuerungsmaßnahmen. Von einer kontinuerlichen Programmbewertung ist das ZDF weit entfernt; zu wenig Klarheit und Übereinstimmung herrscht über die Frage, ob eine kostenund ressourcenintensive Lösung funktional ist. Eine endgültige Entscheidung, ob das Projekt aus der „Erfahrungssammlungsphase“ herauswachsen wird, liegt in der Hand des Intendanten. ${ }^{26 a}$ Die dazu verwendeten Qualitätskriterien sind sehr eng an die Indikatoren des BR angelehnt: „Informationswert", „Unterhaltungswert“, „Zuschauerbindung“, „Genrekompetenz“, „Vitalität/Innovation“, „ästhetische Umsetzung“, „Stellung der Moderation“, „Besonderheitsfaktor“ (USP), ,Werthaltung“, und „Relevanz/Resonanz" sollen grob die Leistungen des ZDF umreissen. Bemerkenswert ist die herausgehobene Stellung der Moderation, deren Qualität zweifelsohne auf die Zuschauerbindung eines Senders rück-

26a So äußerte sich die Leiterin der ZDF-Medienforschung dem Verfasser gegenüber zum schleppenden Verlauf der Projekte. 
wirkt. Ein achtköpfiges Prüfungskomitee vergibt für jede Sendung Schulnoten. Es besteht jeweils aus dem Chefredakteur, weiteren Redakteuren und einer externen Person. Zentrales Moment der Leistungsbewertung beim ZDF ist die Gewichtung. Nach einem komplizierten Schlüssel wird für jede Sendung eigens festgelegt, mit welchem Gewicht die jeweiligen Leistungsindikatoren in die Gesamtbewertung eingehen. Im Falle des Wirtschaftsmagazins "WISO “ geht beispielsweise der Informationswert mit einem deutlich höheren Faktor als der Unterhaltungswert in die Berechnung der Durchschnittsnote ein. ${ }^{27}$

\section{ORF}

Der Österreichische Rundfunk hat seine Leistungsindikatoren für den öffentlich-rechtlichen Rundfunk in vier Dimensionen gegliedert. Das pragmatische Modell umfaßt neben der Quote eine Zuschauernote, die relativen Kosten (Minutenpreis) und das Niveau, über das der öffentlichrechtliche Programmauftrag abgebildet wird. Schwammig wirkt die Operationalisierung des Niveaus: „Wenn es also um die Performance einer Sendung geht, ist unter den Programmverantwortlichen, dem Management und der Medienforschung ein Konsens herzustellen. Dies soll geschehen am besten durch Punktevergabe auf Stimmzetteln in einer Sitzung oder durch interdisziplinäre Rundfrage der Medienforschung bei einer ausreichend großen Zahl von dafür qualifizierten Auskunftspersonen". 28 Berücksichtigt werden soll dabei die "aufklärerische Funktion“, die "soziale Zielgruppenfunktion“, „das künstlerisch-ästhetische Niveau“" und das „Prestige". Jede der vier Größen Quote, Note, Kosten und Niveau wird durch einen Indexwert zwischen eins und fünf ausgedrückt, der auf einem vierdimensionalen Leistungsdiagramm (VDL) abgetragen wird.

\section{Qualitätsinstanzen: Wer darf werten?}

Obwohl in den neunziger Jahren die Auffassung gereift ist, das Publikum als wichtige Bezugsgröße für Qualität zu begreifen, ist die Qualitätsfrage aus der Sicht der Zuschauer noch vergleichsweise selten gestellt worden. ${ }^{29}$ Diesem Defizt will der federführende Bayerische Rundfunk mit

27 Vgl. Teichmann, Andreas: Vom Bildungsprogramm zum Servotainment. Wandel der Wirtschaftsberichterstattung im Fernsehen am Beispiel der Sendung WISO im 2DF. Diplomarbeit am Studiengang Journalistik der Katholischen Universität Eichstätt 1999.

28 Diem 1994 (Anm. 1), S. 70.

29 Zu den seltenen Versuchen zählen: Oehmichen 1993 (Anm. 2); Schenk, Michael/ Gralla, Susanne: Qualitătsfernsehen aus der Sicht des Publikums. Literaturrecherche zum Forschungsstand. In: Media Perspektiven, 1/93 S. 8-15; Schenk, Michael/ 
dem Modell der Programmwert-Kennziffer nun Rechnung tragen. Neben den Zuschauern soll die Qualität noch von einer externen Beobachtergruppe beurteilt werden, überdies sei auch die jeweilige Redaktion in den Evaluationsvorgang einzubinden.

Erfahrungen mit der systematischen Sendungsbeurteilung durch die Zuschauer hat vor allem der $\mathrm{ORF}$ gesammelt. ${ }^{30}$ Dort werden seit vielen Jahren die Reichweiten mit den Beurteilungsnoten einer repräsentativ bestimmten Zuschauergruppe angereichert. Auf einer sechsstufigen Skala halten die Teilnehmer des Tele-Test-Systems ihren Eindruck einer jeden Fernsehsendung fest. Der Nestor der Beurteilungsnoten, Peter Diem, wehrt sich gegen den Vorwurf, die Noten konzentrierten sich zu stark um einen Mittelwert, was ihre Aussagekraft schwäche: „Rund 34 Prozent der Teletest-Noten liegen am und um den Mittelwert, 26 Prozent darunter und 40 Prozent darüber." Für Diem ist das der Beleg, daß die Zuschauer nicht über ein homogen verengtes Urteilsvermögen, dem zwanghaften Trend zum tautologischen Mittelwert, verfügen, sondern in ausreichendem Maße ihre Noten streuen.

Vergeben werden können die Noten entweder über ein Metersystem wie in Österreich oder auch über ein parallel geführtes demoskopisches Instrument, wie der in Großbritannien mittels Umfrage erhobene Appriciation Index (AI). ${ }^{31}$ Allerdings gab es auch in Deutschland schon kontinuierliche Sendungsbeurteilungen durch Fernsehzuschauer. Der 1975 eingestellte "Infratest-Index" war als Forschung für die Programmgestaltung konzipiert worden. ${ }^{32}$ Dieses Bewertungssystem, dessen Skala von +10 bis -10 reichte, gab indes keine Auskunft über die Gründe eines Urteils.

Rossler, Patrick (1990): Rezipientenorientierter Programmvergleich: Ein brauchbares Modell für die Fernsehforschung? In: Media Perspektiven, 12/90 S. 785-791. Im Ausland geht man dagegen mit dem Publikumsgeschmack wesentlich unbefangener um: Vgl. Greenberg, Bradley S./Buselle, Rick: Audience Dimensions of Quality in Situation Comedys and Action Programs. In: Studies of Broadcasting, Nr. 30, März 1994, S. 17-48; dieselben: Television Quality from the Audience Perspective. In; Studies of Broadcasting, Mr. 28, Mărz 1992, S. 157-194; Gunter, Barrie/Winstone, Paul: Television: the public's view 1992. London 1993.

$30 \mathrm{Vgl}$. Diem, Peter: Die Praxis der ORF-Medienforschung. In: Media Perspektiven, 9/93, S. 417-431 sowie Diem 1994 (Anm. 1).

31 Insgesamt arbeitet halb Europa mit zuschauerbezogenen Programmbeurteilungssystemen. Vgl. Diem 1994 (Anm. 1), S. 68.

32 Vgl. Frank, Bernward: Fernsehforschung als Entscheidungshilfe der Programmplanung und -gestaltung. Standortbestimmung und Ausblick. In: Kaase, Max/Schulz, Winfried (Hrsg.): Massenkommunikation. Theorien, Methoden, Befunde. Sonderheft 30 der K.8lner Zeitschrift für Soziologie und Sozialpsychologie. Opladen 1989, S. 270-279, hier S. 275. 
Also Daumen hoch oder runter, wie es seit einigen Jahren die Programmzeitschriften vorexerzieren? Das ist ein wichtiges Argument gegen quantitativ-nummerisch restringierte Bewertungssysteme. Ist es der Inhalt oder die Form, die Gestaltung oder der Stoff, die für Ablehnung oder Zustimmung verantwortlich sind? In Zweifel gezogen wird überdies der Aussagewert eines zu einer einzigen Gesamtnote aggregierten Befundes. Kann ein ohne Indikatoren operierendes Sendungsbeurteilungsverfahren zu zufriedenstellenden Ergebnissen führen? Ein drittes Argument spricht gegen ein derart grob gestricktes und relativ undifferenziertes Beurteilungsverfahren: Gemessen wird durch die Zuschauernote allein das Sendungserlebnis, nicht aber die Güte einer Sendung. Der Zuschauer macht u. U. seiner Enttäuschung über ein verlorenes Fußballspiel, das aus professioneller Sicht brillant in Szene gesetzt sein mag, per Fernbedienung Luft, indem er Tiefstnoten vergibt. Eine Dokumentation über die Greueltaten der Nazis dürtte dem Gros des Publikums dermaßen an die Nieren gehen, daß ein positives Erlebens-Urteil unmöglich wird. Ein Porträt über die beliebte verstorbene Prinzessin Diana ist dagegen geeignet, hohe Werte des Sendungserlebnisses zu erzielen, gleich ob es in puncto Dramaturgie und Regie noch so trivial und vordergründig bleibt. Eine Game-Show, die für Kandidaten und Zuschauer gleichermaßen materielle Gewinne verheißt, hat bessere Chancen, in der Zuschauergunst gut abzuschneiden als die Tagesschau, die ausführlich über einen beginnenden Bürgerkrieg berichtet. Indes „wird der Benotungsunterschied, der zwischen den einzelnen Folgen eines Mehrteilers auftritt, dem Sendungsverantwortlichen immer einen genauen Hinweis auf die Wirkung einzelner Handlungsmuster geben " 33 , etwa bei der "Lindenstraße“. Dessen ungeachtet bleibt die allgemeine Fragwürdigkeit des Publikumsgeschmacks.

Eine weitere externe Evaluationsgröße neben dem Publikum ist der Medienexperte. Er kann sich ausweisen über die praktisch-journalistische Arbeit für eine andere Sendung oder einen anderen Sender, sogar für ein anderes Medium. Auch kann er aus dem Kreis der ausgeschiedenen Journalisten oder der Journalistenausbildung rekrutiert werden beziehungsweise der Zunft der Medienkritiker angehören. Ferner kommen Medienwissenschaftler in Frage, insbesondere dann, wenn sie sich professionell mit journalistischer Qualität beschäftigt haben. Bislang existieren abseits der BR-Pilotstudie kaum Projekte, die solche externen Experten in den Bewertungsprozeß einbezogen haben. Die Vorbehalte gegen Externe sind schon aufgeführt worden. Eine "für diese Branche 
spezifische mimosenhafte Sensibilität gegen Kritik von außen ${ }^{4} 34$ nennt Wolfgang R. Langenbucher diese Bedenken. Dabei kann zur Entkräftung dieser Vorbehalte unterstellt werden, daß Experten über ein ausreichendes Maß an Distanz und Abstraktionsfähigkeit verfügen, um Fernsehsendungen im Hinblick auf ästhetische, dramaturgische, affektiv-emotionale und die Verständlichkeit betreffende Gesichtspunkte zu prüfen. Zur Evaluationsperspektive merkt Langenbucher an: „Eine auf Dauer wirksame kritische Publizität über Medien wird es nur von außen geben, sozusagen von einer Meta-Einrichtung her - oder es wird sie eben nicht geben. 35

Eine vernachlässigte Größe der externen Evaluation bleibt die Fernsehkritik. Ungeachtet der naturbedingten Subjektivität des Kritiker-Urteils, das stark an Wertvorstellungen des Bildungsbürgertums orientiert sein mag, stellt die Programmkritik einen möglichen Maßstab gleichsam kostenlos zur Verfügung. Es wird kaum die einzig mögliche, niemals jedoch eine konsentierte Elle sein, doch ist gerade für öffentlichrechtliche Anbieter eine Richtschnur geeignet, die nicht in unmittelbarer Bodennähe aufgehängt ist. Systematisch und auf jede Sendung bezogen, läßt sich mit der Bezugsgröße TV-und Hörfunkkritik der eigene Anspruch zu einem gewissen Grad abgleichen. Es wird daruiber hinaus aber auch möglich, die Resonanz des einzelnen Senders, der jeweiligen Redaktion zu messen. ${ }^{36}$ Unabhängig von Zuspruch oder Ablehnung, die in einer Programmkritik geäußert werden, ist die Rezension als solche schon ein Indikator für die Folgenhaftigkeit und Relevanz des Programms. Besprochen zu werden, ist per se ein Wert.

Die pro domo wichtigste Beurteilungsinstanz kommt aus den Reihen der eigenen Redaktion. Ob einfaches Redaktionsmitglied, Redaktionsleiter, Abteilungsleiter oder Hauptabteilungsleiter: Der Vorteil liegt im großen Vertrauensvorschuß gegenüber der eigenen Urteils- und Kritikfähigkeit. Auf die Gesamtheit der Journalisten bezogen hat Langenbucher das als "treuherzige Versicherung, daß man aber intern in den Redaktionskonferenzen oder bei sonstigen Gelegenheiten so gnadenlos kritisch miteinander umgehe, wie dies der naive Laie ohnehin nicht könnte ", 37 persifliert. Für den Fall, daß in den Funkhäusern zunächst nur eine BinnenBewertung der Programme vermittelbar ist, sollte gleichwohl langfristig ein Prozeß der allmählichen Auslagerung angestrebt werden. Um den Mitarbeitern den Bewertungsprozeß schmackhaft zu machen, müssen

34 Langenbucher, Wolfgang, R: Zur Idee einer Stiftung Medien-Test. In Rundfunk und Fernsehen, 2/96, S. 240.242, hier S. 240.

35 Ebenda.

36 Vgl. Diem 1994 (Anm. 1), S. 70; Wildberger 1994 (Anm. 25), S. $63 f$. 
diese behutsam und sukzessive an die Unterwerfung durch ein AußenUrteil im Langenbucherschen Sinne herangeführt werden, das die Funktion einer Sendungskritik nach und nach aufnimmt.

Ohnedies sind Eigenbeurteilungen stets der Unaufrichtigkeit verdächtig: Sendungsverantwortliche werden auch im Falle einer unzureichenden Qualität kaum ihre eigenen Produkte schlecht bewerten, weil sie letztlich dafür zur Verantwortung gezogen werden können. Die Umstrukturierung beim DRS hat die personellen Konsequenzen eines mangelnden Erfolges deutlich vor Augen geführt: „Zu divergierenden Einschätzungen kam es zum Teil bei der Bewertung der Reputation. Verantwortliche, die für eine Sendung mit niedrigem Marktanteil zuständig waren, gaben ihren Sendungen tendenziell eine höhere Qualitätsbeurteilung, um zu verhindern, daß die Sendung in den D-Bereich fiel." ${ }^{38}$ Auch bei einer wechselseitigen Beurteilung durch mehrere Redaktionen dürtte die Neigung, daß eine Krähe der anderen kein Auge aushackt, einer um Objektivität bemühten Beurteilung zuwiderlaufen. Zudem gilt analog zum Künstler: Der Journalist ist der schlechteste Interpret seiner Werke.

\section{Das Problem einer angemessenen Gewichtung}

Abseits der Beurteilungs-Instanz bleiben einige weitere Schwierigkeiten der Sendungsbewertung festzuhalten. Die Skalierung ist bei den beschriebenen Projekten noch weitgehend uneinheitlich. Sie reicht von Fünfer-, Sechser- bis zu Zehner- und Elfer-Skalen. Ein Bereich von null bis zehn hat sich dabei als angemessene Lösung herauskristallisiert, vor allem in den west- und mitteleuropäischen Ländern setzt sie sich analog zu anderen sozialwissenschaftlichen Evaluationsprojekten immer weiter durch. Eine differenzierte Maßeinteilung von Null bis Zehn würde auch die Bewertung ein und derselben Sendung durch unterschiedliche Gruppen erleichtern. Ein zur Differenzierung neigendes Expertenurteil wäre durch eine kurze Skala behindert, während eine "Laienbewertung" mit den üblichen Schulnoten auskommen dürfte. Überdies ist auch das Augenmerk auf die Gewichtung der einzelnen Leistungskriterien zu legen. Sie bezieht sich auf zweierlei: Zum einen auf die Einzelindikatoren, die je nach Art des Senders unterschiedlich zueinander ins Verhältnis zu setzen sind. Ein Vollprogramm, das den Ausgleich zwischen öffentlichrechtlichem Programmauftrag und einer gewissen Massenakzeptanz finden muß, wird „Zuschauernähe" und „Nutzwert" der Sendungen einen anderen Stellenwert einräumen als ein minderheitenorientierter Kultur-

38 Wildberger 1994 (Anm. 25), S. 64f. 
sender, für den statt dessen öffentliche "Resonanz", gemessen in Besprechungen, Fernsehpreisen und anderen Renommee-Faktoren, eine große Rolle spielt. Aber auch innerhalb der einzelnen Redaktionen und Sendungen gibt es bedeutsame funktionale Unterschiede in puncto Service- und Nutzwertorientierung, Informationsvermittlung und anderen Programmleistungen. Deshalb macht die sehr differenzierte Gewichtung der Leistungsindikatoren beim ZDF Sinn. "Leute heute" verfolgt andere Absichten als "Aspekte" und muß infolgedessen dem Unterhaltungswert für den Zuschauer einen höheren Stellenwert einräumen. Wichtiger ist dennoch die Frage, wie der Qualitätsfaktor mit den betriebswirtschaftlichen Faktoren vermittelt wird. Es steht einer öffentlich-rechtlichen Rundfunkanstalt nicht gut $\mathrm{zu}$ Gesicht, wenn sie der unter größten Mühen und Schwierigkeiten ermittelten Qualität nur das gleiche Gewicht verleiht, das die Kosten, die Zuschauerakzeptanz und die Verwertbarkeit in die Waagschale legen können - jedenfalls solange, wie daran gedacht wird, mit einer Programmwert-Kennziffer nach außen zu gehen. Für das interne Programmcontrolling im Sinne einer Sendungserfolgskontrolle mag eine Gleichverteilung angemessen sein.

Für einen offensiven Umgang mit den eigenen Leistungen, die mehr denn je der Gesellschaft zu vermitteln sind, ja als ein Instrument der Unternehmens-PR eingesetzt, ist eine andere Gewichtung der Programmwerte vorzunehmen. Es empfiehlt sich gar, nur die Qualitätsquote aus dem Projekt der Programmbewertung herauszulösen und - bei aller sozialwissenschaftlichen Skepsis gegenüber übermäßiger Verdichtung auf einen Index zu reduzieren: Einen simpel zu lesenden Strichcode. Die Einsatzmöglichkeiten reichen im Falle eines konsentierten, von allen öffentlich-.rechtlichen Sendern ratifizierten Modells so weit, daß es sich als institutionalisiertes Gegenargument zu den Quoten aufbauen ließe, um den privaten Sendern diese Parameter aufzuzwingen. Aus dem Münchner Arbeitskreis für öffentlichen Rundfunk (MAR), einer Bürgerinitiative, die sich als kritische Sympathisanten des öffentlichen Rundfunks begreifen, sind deutliche Stimmen zu hören: Sie fordern, daß eine Akzente setzende Öffentlichkeitsarbeit hier einen festen Programmtest für jede Sendung installieren und parallel zum Polit-Barometer ein monatliches Medien-Barometer ausweisen sollte, um die Stärken des öffentlich-rechtlichen Rundfunks herauszustreichen. ${ }^{39}$ Anknüpfungspunkte zur Stiftung Medientest liegen auf der Hand. In den Rundfunkanstalten selbst will man die Qualitätsquoten weit weniger hoch hängen. Der Schwerpunkt liegt noch immer auf einem internen benchmarking.

$39 \mathrm{Vgl}$. Münchner Arbeitskreis offentlicher Rundfunk, unveröffentlichtes Protokoll der Sitzung vom 27. September 1997. 
Ohnehin ist ein öffentlichkeitswirksamer Umgang mit den Qualitätsindikatoren nur dann glaubwürdig und aussichtsreich, wenn er zur Messung der Qualität Außenurteile einholt.

\section{Fazit}

Will man ernsthaft mit der Qualitätsprüfung reüssieren, und hier ist es zunächst egal, ob ein interner oder externer Einsatz beabsichtigt ist, müssen die Praktiker in diesen vergleichsweise aufwendigen Prozeß der Bewertung einbezogen werden. Wichtig ist dabei eine Vermittlung des rechtlich sowie gesellschaftspolitisch formulierten Anspruchs und seiner sozialwissenschaftlichen Operationalisierung mit genuin journalistischen Kriterien der Herstellung. Mit wissenschaftlichen Definitionen der Hochkultur oder der Operationalisierung eines alteuropäischen Anspruchs schreckt man zuständige Redakteure eher ab. Pragmatik ist deshalb gefragt. ZDF-Programmdirektor Markus Schächter nennt das Strategien, "die diese Qualitäts-Semantik greifbarer machen und die abstrakte Rhetorik auf Begriffe des alltäglicheren Arbeitens runterbrechen sollen." ${ }^{40}$ Ein politisches Magazin hat dann einen hohen Informations. wert, wenn die Themen so präsentiert werden, daß neben den vier journalistischen Fragewörtern WER, WANN, WO und WIE auch noch das WARUM beantwortet wird. Nur auf diesem Weg sind Strukturen und Zusammenhänge darzustellen und Hintergründe zu vermitteln. Eine investigative Reportage ist dann handwerklich sauber produziert, wenn bei der Informationssuche mindestens zwei unabhängige verläßliche Quellen vom Reporter befragt wurden, wenn im Konfliktfall alle Seiten (audiatur et altera pars) mit ihren Positionen, Sichtweisen und Stellungnahmen präsent sind und wenn schließlich ein roter Faden erkennbar ist. Das sind Kategorien, die in der Lebenswelt der Praktiker vorkommen. Eine Diskussionssendung ist dann gesellschaftlich relevant, wenn sie vom Einzelfall abstrahieren kann und die soziale Folgenhaftigkeit eines ursprünglich singulären Problems erörtert.

Unabhängig davon, wer letztlich die Beurteilung einer Sendung vornimmt, muß das Ziel der Vorüberlegungen zur Sendungsbewertung sein, eine offene Liste der qualitätsunterstützenden Spezifikationen herzustellen. Für alle Parameter ist deshalb festzulegen, wie eine optimale Sen-

40 Markus Schächter zitiert in: Qualitatsfermsehen hat seinen Preis. In: Grimme $1 / 1998$, S. 6.8 . 
dung ungefähr auszusehen hätte. Folglich ist ein Idealzustand als Maßstab, als wünschenswerte Zielvorstellung $z \mathfrak{u}$ formulieren.

Am Schluß stellt sich die Frage, ob einer großen oder kleinen Lösung der Vorrang einzuräumen ist. Hiebei ist an die Funktion der Sendungsbewertung zu denken. $\mathrm{Zu}$ berücksichtigen ist, ob ein kontinuierliches $\mathrm{Be}$ wertungsmodell angestrebt wird oder, ob das Meßinstrument nur dem stichprobenartigen Ad-hoc-Einsatz dient. Die Beantwortung dieser Frage ist ebenso richtungsweisend wie die Bestimmung des Operationsgebietes. Für den internen Einsatz dürfte ein pragmatisches Modell mit einem überschaubaren, zumutbaren Katalog von Leistungskriterien ausreichen, das die Qualität nur über die Binnenperspektive der Macher bestimmt. Diese Sicht könnte unsystematisch angreichert werden durch vereinzelte Zuschauerbefragungen und die Auswertung von Pressestimmen und Fernsehkritiken, die ohnehin in der Öffentlichkeitsarbeit gesammelt werden. Wie eine große Lösung auszusehen hätte, wurde in der Studie grob skizziert: Alle am Kommunikationsprozeß beteiligten Akteure müßten systematisch Urteile abgeben, so daß alle Bewertungsdimensionen abgedeckt wären. Ein solches komplexes Modell bedarf nicht nur aller denkbaren mehrdimensional verknüpften Faktoren, es müßte auch jeden Indikator nach Maßgabe der festgelegten relevanten Spezifikationen explizieren, so daß jederzeit eine intersubjektive Nachvollziehbarkeit gewährleistet bleibt, die wissenschaftlich einklagbar ist. Offengelegt werden muß dann auch die Gewichtung der Faktoren, die nach Maßgabe des Sendeplatzprofils vorzunehmen ist.

Die wichtigste Voraussetzung, um mit den Qualitätsindikatoren in die Offensive gehen zu können und die Eigenschaften des öffentlich-rechtlichen Rundfunks im Bewußtsein der Öffentlichkeit wirksam zu verankern, zielt aber nach innen. Es müssen den Mitarbeitern in den Sendern die Chancen erläutert werden, die in der Veröffentlichung der Sendungsqualität liegen. Das Wuchern mit dem Pfund "Qualität" enthält - weit gedacht - auch die Option der Arbeitsplatzsicherungsmaßnahme. Wenn ich unter der Voraussetzung, von der eigenen Leistung überzeugt zu sein, die Qualität meiner Arbeit bewerten und kontrollieren lasse, ist das nicht nur ein Ausweis von Selbstbewußtsein, sondern auch ein Investition in die Sicherung meines Arbeitsplatzes. Dieser Gedanke ist für viele öffentlich-rechtliche Angestellte vermutlich besorgniserregend radikal und dem eigenen Denken fremd, auf lange Sicht muß er aber in den Häusern vermittelt werden. 